\title{
Medical Robotics
}

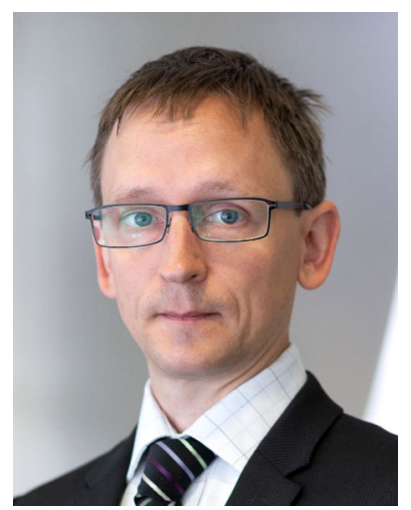

Daniel S. Elson

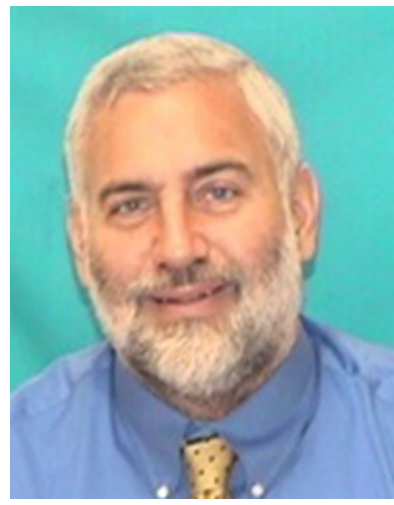

Kevin Cleary

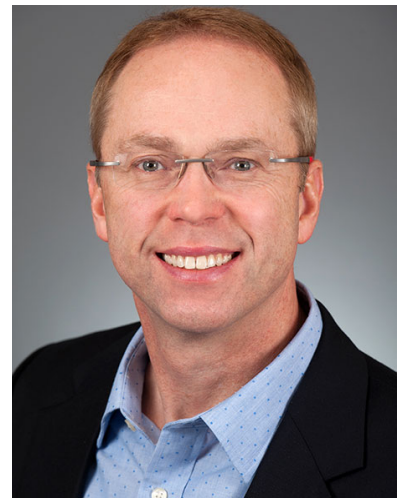

Pierre Dupont

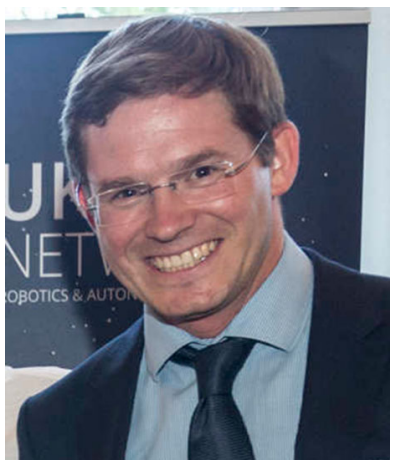

Robert Merrifield

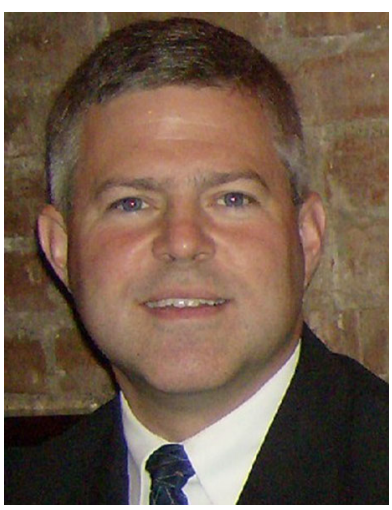

Cameron Riviere

Medical robotics encompasses surgical, therapeutic and rehabilitative devices that are changing medicine and healthcare. Although the field of medical robotics predates Intuitive's da Vinci by more than a decade, it was the clinical and commercial achievements of that system that brought medical robotics to widespread patient and public attention. It is now more than 15 years since the robot began to be used for laparoscopic prostatectomy. ${ }^{1}$ Since then, research in the field has advanced tremendously due to various technological breakthroughs. Over the last few years, there has been a surge in commercial activities in medical robotics, led both by traditional medical device and technology companies as well as new start-ups. This special issue has been commissioned to capture some 
of the latest research being carried out by these multidisciplinary bioengineering teams and to showcase how some of these advances can impact clinical care.

The Hamlyn Symposium on Medical Robotics is an annual event that celebrated its 10th edition between 25 and 28 June 2017. This event attracts researchers from around the world who present their work on novel robotic and interventional technologies - including imaging, diagnostics and image-guidance - with a strong focus on clinical translation and clinical studies. It is supported by an international programme committee and robust peer review and selection process. Some of the papers invited for this special issue have been selected from those presented at the symposium, together with some additional papers invited from outstanding researchers. All the papers have undergone the full ABME peer review process.

Novel robotic instruments are presented in several papers; for instance a device $2 \mathrm{~mm}$ in diameter with three degrees of freedom is developed using nitinol tubing. ${ }^{8}$ A handheld tool is proposed that can help with procedures such as petroclival meningioma resection, which require microresection to avoid damage to surrounding brain and blood vessels. ${ }^{16}$ This was achieved using deep learning for segmentation of blood vessels from microscopy images and it was shown that the system could help in safe vessel avoidance. Robots for neurosurgery can be used for better placement of needles, catheters, probes, or electrodes, but are often large, bulky and complex. In Li et al. ${ }^{13}$ a lightweight parallel mechanism that can attach to the skull is designed and prototyped. We also include a review article that provides insight into the latest developments in MRIcompatible robots for needle-based interventions. ${ }^{17}$

The flexibility of robotic instruments is important for improving dexterity and enabling new applications, and continuum robots that have segments that are capable of continuous bending are emerging using various technologies. One example for potential use in nephrectomy ${ }^{3}$ is a flexible, robotic, variable-stiffness port that can allow a single-incision approach by improving access over the manual instruments. Concentric tube robots are an alternative continuum approach and in Chikhaoui et al..$^{7}$ a biocompatible conducting polymer is presented and evaluated. A patient-specific concentric tube robot is also designed from preoperative images through to physical implementation and evaluation. ${ }^{18}$ The concept of soft robotics was investigated for robotic cardiac assist devices that attach to the external surface of the heart, and different interface materials were evaluated in an animal model. ${ }^{12}$

The interaction between the robot and the operator is a crucial consideration. The issue contains several papers that aim to improve decision support and monitoring of the intervention through improved sensing or imaging. For example, Manbachi et al. ${ }^{15}$ assess a method for automatic labeling of vertebrae in intraoperative radiographs in the operating room during spine surgery. In Cheng et al. ${ }^{6}$ a smart venous entry indicator is developed that uses bio-impedance measured at the tip of a peripheral intravenous catheterization device to improve the ability of test subjects to successfully catheterize a baby arm phantom. A method to ensure a safe intraoperative margin between a drill trajectory and the facial nerve using monopolar and bipolar stimulation threshold measurements during robotic cochlea implantations has been validated during a first-in-man trial. ${ }^{4}$ Image guidance may be used to improve targeting accuracy during needle biopsies although it may not account for target or needle displacement, an issue that is addressed by Wartenberg et al. $^{21}$ An augmented reality system is proposed for computer assisted orthopedic surgery that makes use of a depth sensitive camera for registration purposes, accounting for and avoiding obstacles. ${ }^{14}$ Robotic hand-eye calibration is important when moving towards more autonomous actuation, and an improved algorithm to achieve this is proposed and evaluated based on the adjoint transformation of twist motions. ${ }^{19}$ In a twist on the theme of this section, in Singh et al., ${ }^{20}$ the effect that manual and robotic suturing tasks have on the prefrontal cortex is assessed with near-infrared spectroscopy. The insight provided by the end-users - surgeons in this case - is a key to successful design of robotic systems and workflows, and it has been shown that recovery time, operation time and cost can all be reduced for minimally invasive reduction of intra-articular fractures. ${ }^{9}$

One of the recent additions to the Hamlyn Symposium is the Surgical Robot Challenge, an international competition to develop low-cost robot-assisted surgical and diagnostic devices. The emphasis is on creating new concepts for affordable systems especially with potential for applications in the developing world. Four of the papers in this special issue are based on the prize-winning entrants in the competition, namely, 'Toward Semi-Autonomous Cryoablation of Kidney Tumors via Model-Independent Deformable Tissue Manipulation Technique', 'The i'Snake Robotic Platform for Endoscopic Surgery', 'In-Human-Robot-Assisted Retinal Vein Cannulation, a World First', ${ }^{10}$ and 'Stormram 4: An MR Safe Robotic System for Breast Biopsy'. ${ }^{11}$

We hope that these papers will be of interest and value to the ABME community. We thank all of the contributors, reviewers and ABME editorial staff for their efforts in putting this special issue together. 


\section{REFERENCES}

${ }^{1}$ Ahlering, T. E., D. Skarecky, D. Lee, and R. V. Clayman. Successful transfer of open surgical skills to a laparoscopic environment using a robotic interface: initial experience with laparoscopic radical prostatectomy. J. Urol. 170(5):1738-1741, 2003.

${ }^{2}$ Alambeigi, F., Z. Wang, Y.-H. Liu, R. H. Taylor, and M. Armand. Toward semi-autonomous cryoablation of kidney tumors via model-independent deformable tissue manipulation technique. Ann. Biomed. Eng. 2018. https:// doi.org/10.1007/s10439-018-2074-y.

${ }^{3}$ Amanov, E., T.-D. Nguyen, S. Markmann, F. Imkamp, and J. Burgner-Kahrs. Toward a flexible variable stiffness endoport for single-site partial nephrectomy. Ann. Biomed. Eng. 2018. https://doi.org/10.1007/s10439-018-2060-4.

${ }^{4}$ Ansó, J., O. Scheidegger, W. Wimmer, K. Gavaghan, N. Gerber, D. Schneider, J. Hermann, C. Rathgeb, C. Dür, K. M. Rösler, G. Mantokoudis, M. Caversaccio, and S. Weber. Neuromonitoring during robotic cochlear implantation: initial clinical experience. Ann. Biomed. Eng. 2018. https://doi.org/10.1007/s10439-018-2094-7.

${ }^{5}$ Berthet-Rayne, P., G. Gras, K. Leibrandt, P. Wisanuvej, A. Schmitz, C. A. Seneci, and G.-Z. Yang. The $i^{2}$ snake robotic platform for endoscopic surgery. Ann. Biomed. Eng. 2018. https://doi.org/10.1007/s10439-018-2066-y.

${ }^{6}$ Cheng, Z., B. L. Davies, D. G. Caldwell, and L. S. Mattos. A new venous entry detection method based on electrical bio-impedance sensing. Ann. Biomed. Eng. 2018. https:// doi.org/10.1007/s10439-018-2025-7.

${ }^{7}$ Chikhaoui, M. T., A. Benouhiba, P. Rougeot, K. Rabenorosoa, M. Ouisse, and N. Andreff. Developments and control of biocompatible conducting polymer for intracorporeal continuum robots. Ann. Biomed. Eng. 2018. https://doi.org/10.1007/s10439-018-2038-2.

${ }^{8}$ Francis, P., K. W. Eastwood, V. Bodani, T. Looi, and J. M. Drake. Design, modelling and teleoperation of a $2 \mathrm{~mm}$ diameter compliant instrument for the da vinci platform. Ann. Biomed. Eng. 2018. https://doi.org/10.1007/s10439018-2036-4.

${ }^{9}$ Georgilas, I., G. Dagnino, P. Tarassoli, R. Atkins, and S. Dogramadzi. Robot-assisted fracture surgery: surgical requirements and system design. Ann. Biomed. Eng. 2018. https://doi.org/10.1007/s10439-018-2005-y.

${ }^{10}$ Gijbels, A., J. Smits, L. Schoevaerdts, K. Willekens, E. B. Vander Poorten, P. Stalmans, and D. Reynaerts. In-human robot-assisted retinal vein cannulation, a world first. Ann. Biomed. Eng. 2018. https://doi.org/10.1007/s10439-0182053-3.

${ }^{11}$ Groenhuis, V., F. J. Siepel, J. Veltman, J. K. van Zandwijk, and S. Stramigioli. Stormram 4: an MR safe robotic system for breast biopsy. Ann. Biomed. Eng. 2018. https://doi.org/ 10.1007/s10439-018-2051-5.

${ }^{12}$ Horvath, M. A., C. E. Varela, E. B. Dolan, W. Whyte, D. S. Monahan, C. J. Payne, I. A. Wamala, N. V. Vasilyev, F. A. Pigula, D. J. Mooney, C. J. Walsh, G. P. Duffy, and E. T. Roche. Towards alternative approaches for coupling of a soft robotic sleeve to the heart. Ann. Biomed. Eng. 2018. https://doi.org/10.1007/s10439-018-2046-2.

${ }^{13} \mathrm{Li}, \mathrm{C}$., N. K. K. King, and H. Ren. A skull-mounted robot with a compact and lightweight parallel mechanism for positioning in minimally invasive neurosurgery. Ann.
Biomed. Eng. 2018. https://doi.org/10.1007/s10439-0182037-3.

${ }^{14}$ Liu, H. E. Auvinet, J. Giles, and F. Rodriguez y Baena. Augmented reality based navigation for computer assisted hip resurfacing: a proof of concept study. Ann. Biomed. Eng. 2018. https://doi.org/10.1007/s10439-018-2055-1.

${ }^{15}$ Manbachi, A., T. De Silva, A. Uneri, M. Jacobson, J. Goerres, M. Ketcha, R. Han, N. Aygun, D. Thompson, X. Ye, S. Vogt, G. Kleinszig, C. Molina, R. Iyer, T. GarzonMuvdi, M. R. Raber, M. Groves, J.-P. Wolinsky, and J. H. Siewerdsen. Clinical translation of the levelcheck decision support algorithm for target localization in spine surgery. Ann. Biomed. Eng. 2018. https://doi.org/10.1007/s10439018-2099-2.

${ }^{16}$ Moccia, S., S. Foti, A. Routray, F. Prudente, A. Perin, R. F. Sekula, L. S. Mattos, J. R. Balzer, W. Fellows-Mayle, E. De Momi, and C. N. Riviere. Toward improving safety in neurosurgery with an active handheld instrument. Ann. Biomed. Eng. 2018. https://doi.org/10.1007/s10439-0182091-x.

${ }^{17}$ Monfaredi, R., K. Cleary, and K. Sharma. MRI robots for needle-based interventions: systems and technology. Ann. Biomed. Eng. 2018. https://doi.org/10.1007/s10439-0182075-x.

${ }^{18}$ Morimoto, T. K., J. D. Greer, E. W. Hawkes, M. H. Hsieh, and A. M. Okamura. Toward the design of personalized continuum surgical robots. Ann. Biomed. Eng. 2018. https://doi.org/10.1007/s10439-018-2062-2.

${ }^{19}$ Pachtrachai, K., F. Vasconcelos, F. Chadebecq, M. Allan, S. Hailes, V. Pawar, and D. Stoyanov. Adjoint transformation algorithm for hand-eye calibration with applications in robotic assisted surgery. Ann. Biomed. Eng. 2018. https://doi.org/10.1007/s10439-018-2097-4.

${ }^{20}$ Singh, H., H. N. Modi, S. Ranjan, J. W. R. Dilley, D. Airantzis, G.-Z. Yang, A. Darzi, and D. R. Leff. Robotic surgery improves technical performance and enhances prefrontal activation during high temporal demand. Ann. Biomed. Eng. 2018. https://doi.org/10.1007/s10439-0182049-z.

${ }^{21}$ Wartenberg, M., J. Schornak, K. Gandomi, P. Carvalho, C. Nycz, N. Patel, I. Iordachita, C. Tempany, N. Hata, J. Tokuda, and G. S. Fischer. Closed-loop active compensation for needle deflection and target shift during cooperatively controlled robotic needle insertion. Ann. Biomed. Eng. 2018. https://doi.org/10.1007/s10439-018-2070-2.

\section{DANIEL S. ELSON}

Hamlyn Centre for Robotic Surgery and

Department of Surgery and Cancer,

Imperial College London,

London, UK

Electronic mail: daniel.elson@imperial.ac.uk

\section{Kevin Cleary}

Bioengineering Initiative, Children's National

Medical Center,

Washington, USA 
Pierre Dupont

Pediatric Cardiac Bioengineering,

Boston Children's Hospital,

Boston, USA

\section{Robert MERrifield}

Hamlyn Centre for Robotic Surgery and Department of Computing,

Imperial College London,

London, UK

\section{Cameron Riviere}

The Robotics Institute, Carnegie Mellon University. Pittsburgh, USA 